Herwig, A. Schmitt, J. Freund, T. Elge, R. Mott et al. 1998. Comparative gene expression profiling by oligonucleotide fingerprinting. Nucleic Acids Res. 26:2216-2223.

13.Radelof, U., S. Hennig, P. Seranski, M. Steinfath, J. Ramser, R. Reinhardt, A. Poustka, F. Francis et al. 1998. Preselection of shotgun clones by oligonucleotide fingerprinting: an efficient and high throughput strategy to reduce redundancy in large-scale sequencing projects. Nucleic Acids Res. 26:53585364 .

14.Schena, M., D. Shalon, R. Heller, A. Chai, P.O. Brown, and R.W. Davis. 1996. Parallel human genome analysis: microarray-based expression monitoring of 1000 genes. Proc. Natl. Acad. Sci. USA 93:10614-10619.

15.Speicher, M.R., S.G. Ballard, and D.C. Ward. 1996. Karyotyping human chromosomes by combinatorial multi-fluor FISH. Nat. Genet. 12:368-375.

16. Wittung, P., P.E. Nielsen, O. Buchardt, M. Egholm, and B. Norden. 1994. DNA-like double helix formed by peptide nucleic acid. Nature 368:561-563.

17.Wodicka, L., H. Dong, M. Mittmann, M.-H. Ho, and D.J. Lockhart. 1997. Genome-wide expression monitoring in Saccharomyces cerevisiae. Nat. Biotechnol. 15:1359-1367.

This work has been financed by the Max Planck Society. Address correspondence to Dr. Anna Guerasimova, Max-Planck-Institute for Molecular Genetics, Ihnestr. 73, Berlin, D-14193, Germany.e-mail: guer@ molgen.mpg.de

Received 24 July 2000; accepted 15 March 2001.

A. Guerasimova, L. Nyarsik, I. Girnus, M. Steinfath, W. Wruck, H. Griffiths,

R. Herwig, C. Wierling, J. O'Brien ${ }^{1}$, H. Eickhoff, H. Lehrach, and U. Radelof

Max-Planck-Institute for Molecular Genetics

Berlin, Germany

${ }^{1}$ Royal College of Surgeons in Ireland

Dublin, Ireland
For reprints of this or any other article, contact Reprints@BioTechniques.com

\section{Colorimetric Assay to Quantify Macromolecule Diffusion across Endo- thelial Monolayers}

BioTechniques 31:495-501 (September 2001)

\section{ABSTRACT}

Endothelial "capillary leak", the loss of vascular integrity in response to noxious stimuli, is characterized by extravasation of protein-rich fluid from capillary lumen into surrounding tissue interstitium. This increase in vascular permeability, in response to inflammatory mediators, correlates with endothelial cell contraction and the formation of intercellular gaps within the monolayer. However, in vivo assessment of paracellular solute flow between endothelial cells may be complicated by multiple uncontrolled parameters. In vitro examinations of endothelial barrier leak have relied on electrical impedence or macromolecule diffusion techniques to determine the details pertinent to capillary barrier function. In this report, a simple, sensitive, nonradioactive, colorimetric assay to quantify the leak of a labeled protein marker across endothelial monolayers is described. This procedure avoids the hazards of radioisotope labels and the technical limitations of electrical resistance technology.

\section{INTRODUCTION}

In vitro quantification of macromolecular leak across endothelial cell monolayers grown on semi-permeable membranes has facilitated the analysis of vascular integrity in response to isolated inflammatory mediators and pharmaceutical agents. Numerous investigations of vascular endothelial physiology, cell-cell interaction, and resultant endothelial capillary leak have been performed utilizing this experimental model. Current methods for detecting endothelial cell monolayer leak or "permeability" generally rely on one of two methods: measurement of transmonolayer electrical impedence or measurement of labeled macromolecular diffusion across cell monolayers. 
Assessing changes in monolayer integrity by transendothelial electrical resistance (TEER) assumes that minute alterations occur in the size and number of intercellular gaps in response to stimulation (7). Compared with the remarkable decrease in impedence observed when tightly adherent epithelial cell monolayers are stimulated, the difference in TEER between stimulated and unstimulated endothelial cell monolayers is very narrow, making significant measurements difficult with this cell type. Furthermore, as TEER measures trans-monolayer impedence to electrical current only, the physical and chemical properties of macromolecular solutes that alter diffusion of these large molecules across monolayers are not accounted for by this technique. Therefore, the use of radioisotope- or fluorescence-labeled [iodine-125, fluorescein isothiocyanate (FITC), isocyanide, etc.] marker proteins may provide a more physiologically accurate model of solute leak (2). However, labeled reagents, particularly solutes labeled with nonradioactive fluorophore compounds, often lack the sensitivity to demonstrate subtle changes in monolayer permeability that are due to poor specific activity (fluorescence/mg protein) or fluorophore instability. The specific advantage of biotinylation of marker proteins over FITC labeling as a means of quantifying monolayer leak is 2 -fold. FITC fluorophores are routinely conjugated with antibodies and other proteins for qualitative purposes, such as in immunohistological staining. Fluorophore activity degrades upon exposure to oxidizing compounds, metal ions, and light, diminishing the sensitivity of these compounds to quantify monolayer leak. Avidin binds biotin with extremely high affinity $\left(\mathrm{k}_{\mathrm{d}}=10^{-15}\right.$ $\mathrm{M}^{-1}$ ) and specificity. These compounds are stable and exhibit little decrease in affinity for one another, even under harsh conditions. Activity of the detection agent, horseradish peroxidase (HRP)-labeled streptavidin, would be uniform among all wells of a given assay. A second disadvantage of fluorophore-labeled solutes is the equipment required for their detection. Fluorescent molecules, including FITC, require excitation at specific wavelengths to emit light. Excitation- capable plate readers are expensive and possibly unavailable, while the use of the common single-cuvette fluorescence spectrophotometers would be quite tedious. Detection with HRP-labeled streptavidin provides a simple, reproducible means to quantify biotin colorimetrically that is within the capability of most ELISA plate readers.

The use of radiolabeled markers, while enhancing sensitivity, is complicated by the need for specific precautions for safe handling and storage. Furthermore, because of their short half-life, isotopes are unsuitable for long-term storage. Enzymatic markers such as HRP have been used to exam ine macromolecule diffusion across monolayers by tracking supernatant HRP activity (6). Unfortunately, $\mathrm{pH}$, temperature, and serum constituents may affect the activity of enzymatic markers and limit their suitability for many investigations.

In this report, we describe a nonradioactive, sensitive, rapid, and reproducible method of assessing in vitro cellular monolayer "leak" that utilizes a biotinylated marker [bovine serum albumin (BSA)] and a plate-based, colorimetric analysis detection system that has been developed for use with several human and animal endothelial cell types.

\section{MATERIALS AND METHODS}

Unless otherwise indicated, all reagents were from Sigma-Aldrich (St. Louis, MO, USA).

\section{Biotin Labeling of Marker Proteins}

Fraction V BSA was biotinylated through primary amine coupling by the method of Hofmann et al. (1). Follow ing dissolution of $10 \mathrm{mg}$ biotinamidocaproate $\mathrm{N}$-hydroxysuccinimide in 2.54 mL N,N-dimethyl formamide, a 20 -fold molar excess was added to a $20 \mathrm{mg} / \mathrm{mL}$ solution of BSA with gentle mixing. The reaction was allowed to proceed in the dark for $2 \mathrm{~h}$ with intermittent gentle agitation. Biotinylated BSA was separated from unbound biotin through dialysis (10000 MWCO tubing, SpectraPor $^{\mathrm{TM}}$; Fisher Scientific, Pittsburgh, PA, USA) against a $500 \times$ volume of PBS at $4^{\circ} \mathrm{C}$. Four changes of PBS were re- quired to remove all uncoupled biotin. Employing an extinction coefficient of 0.67 , absorption at $280 \mathrm{~nm}$ was used to determine the concentration of the conjugate. The absence of peaks at wavelengths greater than $300 \mathrm{~nm}$ during absorptive spectrophotometry established that the sample was free of low molecular weight contaminants.

Biotin labeling was confirmed by dot blot. Serial dilutions of stock BSAbiotin were prepared in log increments ( $1 \mathrm{mg} / \mathrm{mL}$ to $1 \mathrm{ng} / \mathrm{mL}$ ). Two microliters of each were spotted onto $0.2-\mu \mathrm{m}$ nitrocellulose membrane (Pierce Chemical, Rockford, IL, USA) and allowed to dry. Nitrocellulose was blocked with $2 \%$ BSA (unlabeled) for $1 \mathrm{~h}$ at room tem perature, followed by the addition of HRP-labeled-streptavidin at 1:5000 dilution (KPL, Gaithersburg, MD, USA). After $1 \mathrm{~h}$, the non-bound streptavidin was removed in $100 \mathrm{~mL}$ PBS containing $0.05 \%$ Tween ${ }^{\circledR} 20$ (PBS-T) with three 10-min washes. Membranebound BSA-biotin was detected with carbazole-peroxide substrate (AEC substrate; Pierce Chemical) for $10 \mathrm{~min}$ at room temperature. Conjugate lots demonstrating sufficient specific activity $(\mathrm{AB} . / \mathrm{mg} \mathrm{BSA})$ were frozen at $-80^{\circ} \mathrm{C}$ in $30 \mathrm{mg} / \mathrm{mL}$ aliquots for future use.

\section{Assessment of Endothelial Monolayer Integrity}

Human umbilical vein endothelial cells (HUVEC; Clonetics, La Jolla, CA, USA) were plated on $0.4-\mu$ m pore semi-permeable polyester membranes (Costar, Corning, NY, USA) at a concentration of $5 \times 10^{4}$ cells $/ \mathrm{mL}$ in EGM2-mv media (Clonetics). Mem branes were placed into co-culture wells, dividing the well into upper and lower chambers. Lower compartments were filled with sufficient media to cover the underside of the membrane $(1.25 \mathrm{~mL})$. Cells were grown to confluence (5-7 days) before use, with media changes every second day.

Endothelial growth medium contains trace amounts of biotin as a nutrient; as such, cells were gently washed twice immediately before experimental treatments with Hank's Balanced Salt Solution (HBSS) containing $0.5 \%$ fetal bovine serum (FBS) and $10 \mathrm{mM}$ $\mathrm{N}-$-'(2-Hydroxyethyl) piperazine-N-(2- 


\section{Short Technical Reports}

ethanesulfonic acid) (HEPES). The removal of media and washes before experimental treatment dramatically reduced background signal in the biotin detection portion of this assay. The HBSS/FBS was used as a buffer solution capable of supporting normal cell metabolism for the duration of the assay.

BSA-biotin was diluted in sufficient volume of HEPES-buffered HBSS/FBS to a final concentration of $300 \mu \mathrm{g} / \mathrm{mL}$. Addition of BSA-biotin to stock HEPES-buffered HBSS/FBS minimized inter-well variation due to pipetting errors. Aliquots of this solution were utilized to generate of a standard curve. BSA-biotin solution $(500 \mu \mathrm{L})$ was added to the upper chamber of each well, while $1.25 \mathrm{~mL}$ BSA-biotin-free HBSS/FBS buffer were added to each lower chamber. Simultaneously, experimental reagents (to be assessed for their effect on endothelial integrity) were added to the upper chamber of culture wells in minimal volume $(5 \mu \mathrm{L} 100 \times$ stock). A standard reagent, $100 \mu \mathrm{M}$ human thrombin, was included in separate wells as the positive control for each plate. Identical amounts of HBSS buffer were added to control wells to avoid hydrostatic or oncotic imbalances related to buffer. At $30 \mathrm{~min}, 2$, 4, and 24 $\mathrm{h}, 50 \mu \mathrm{L}$ samples were removed from lower chambers and stored at $4{ }^{\circ} \mathrm{C}$.

\section{Detection of Biotin-labeled Marker Proteins}

Lower chamber supernatant aliquots and biotin-BSA standards were diluted $10 \times$ and $100 \times$ in fresh bicarbonate sensitizing buffer $(293 \mathrm{mg} \mathrm{NaHCO}+159$ $\mathrm{mg} \mathrm{Na} \mathrm{CO}_{3}+100 \mathrm{~mL}$ double-distilled water, $\mathrm{pH}$ 9.0) and added to individual wells of a 96-well, high protein-binding plate (Immulon $4{ }^{\circledR}$; Dynex Technolo- gies, Chantilly, VA, USA). Plates were covered and incubated overnight at $4{ }^{\circ} \mathrm{C}$. Wells were washed with $0.05 \%$ PBS-T and then blocked with $2 \%$ unlabeled BSA (in PBS-T) for $2 \mathrm{~h}$ at room temperature. Biotin-labeled BSA was detected in each well by the addition of HRP-labeled streptavidin (1:5000 in 1\% BSA/ PBS-T) for $1 \mathrm{~h}$ at room temperature. Plates were washed four times in PBS$\mathrm{T}$ before development. Ophenylediamine $(10 \mathrm{mg})$ was dissolved in substrate buffer $\left(10 \mathrm{~mL} 0.2 \mathrm{M} \mathrm{Na}_{2} \mathrm{HPO}_{4}+\right.$ $10 \mathrm{~mL} 0.1 \mathrm{M}$ citric acid $+10 \mu \mathrm{L} 30 \%$ $\mathrm{H}_{2} \mathrm{O}_{2}$ ), and $100 \mu \mathrm{L}$ were added to each well. Development was halted with addition of $100 \mu \mathrm{L} 0.2 \mathrm{M}$ sulfuric acid, and the plate was read at $490 \mathrm{~nm}$ on a Revelation MRX ${ }^{\circledR}$ plate reader (Dynex Technologies). BSA-biotin concentrations in supernatant samples were determined by comparison to a simultaneously generated standard curve. 


\section{Short Technical Reports}

\section{RESULTS AND DISCUSSION}

This assay was designed to examine in vitro endothelial changes that may contribute to capillary barrier dysfunction in vivo. Using this method, $100 \mu \mathrm{M}$ human thrombin were shown to induce a rapid increase in the diffusion of BSAbiotin across HUVEC monolayers. Figure 1 demonstrates detection of lower chamber BSA-biotin $(\mathrm{pg} / \mathrm{mL})$ versus untreated control wells at $30 \mathrm{~min}$ following exposure to $100 \mu \mathrm{M}$ thrombin, $10 \mu \mathrm{M}$ calcimycin, or $50 \mu \mathrm{M} \mathrm{H}_{2} \mathrm{O}_{2}$. We have successfully utilized this detection system with several primary and transformed micro- and macrovascular human endothelial cell types [HUVEC; lung microvascular (HMVEC-L); coronary artery (HCAEC); dermal microvascular (HMEC.1); bovine pulmonary microvascular (PMEC)] and a variety of endothelial capillary leak-inducing and inhibiting reagents (3). These assays were carried out in HBSS/0.5\% FBS. Microvascular endothelial cells grow poorly in serum-free conditions without extensive subcloning and gradual reduction of serum content. Our system allowed the use of early passage primary endothelial cells of numerous vascular origins. The low concentration of FBS $(0.5 \%)$ in the assay buffer prevented detachment and maintained monolayer integrity. As the luminal aspect of endothelial cells is exposed to serum in vivo, serum exposure of in vitro buffers are unlikely to present an abnormal physiological condition. The trans-well membrane alone provided a degree of resistance to solute flux, though permeability was greatly increased by the presence of confluent endothelial cell monolayers (Figure 2).

Albumin, the major soluble circulatory protein in vivo, which possesses 39 accessible tyrosine residues for biotinylation, is inexpensive and stable. Evans Blue-labeled BSA (EBA) has also been described for in vitro quantification of capillary leak. Our examination of EBA as a marker for quantifying human endothelial monolayer permeability detected a lower limit of detection in the range of 10-100 $\mu \mathrm{g} / \mathrm{mL}$ well-labeled albumin. Biotinylation permits the detection of albumin at $10^{5}$-fold lower concentrations than Evans Blue labeling. Evans Blue Dye

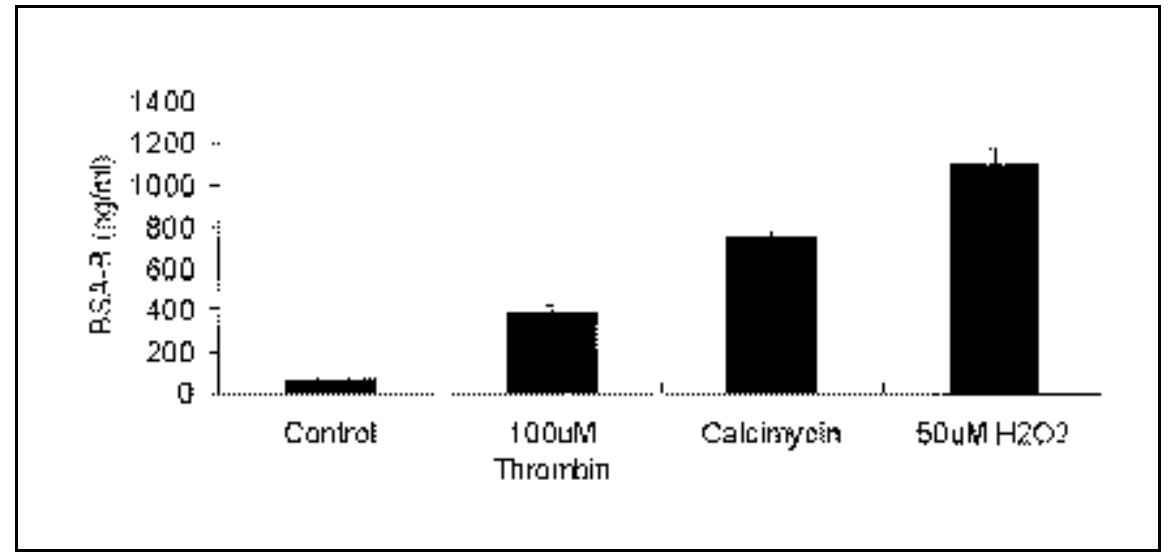

Figure 1. Diffusion of biotin-BSA across HUVEC monolayers grown on a 0.4- $\mu \mathrm{m}$ semi-permeable membrane. Human thrombin $(100 \mu \mathrm{M}), 10 \mu \mathrm{M}$ calcimycin, or $50 \mu \mathrm{M} \mathrm{H} \mathrm{H}_{2}$, and biotin-BSA in HEPES-buffered HBSS/FBS were added to upper chamber wells at time zero, with equivalent volumes of HEPES-buffered HBSS/FBS added to Control group. Lower chamber supernatants $(50 \mu \mathrm{L})$ were assessed at $2 \mathrm{~h}$ and assayed for biotin-BSA using a plate-based detection method.

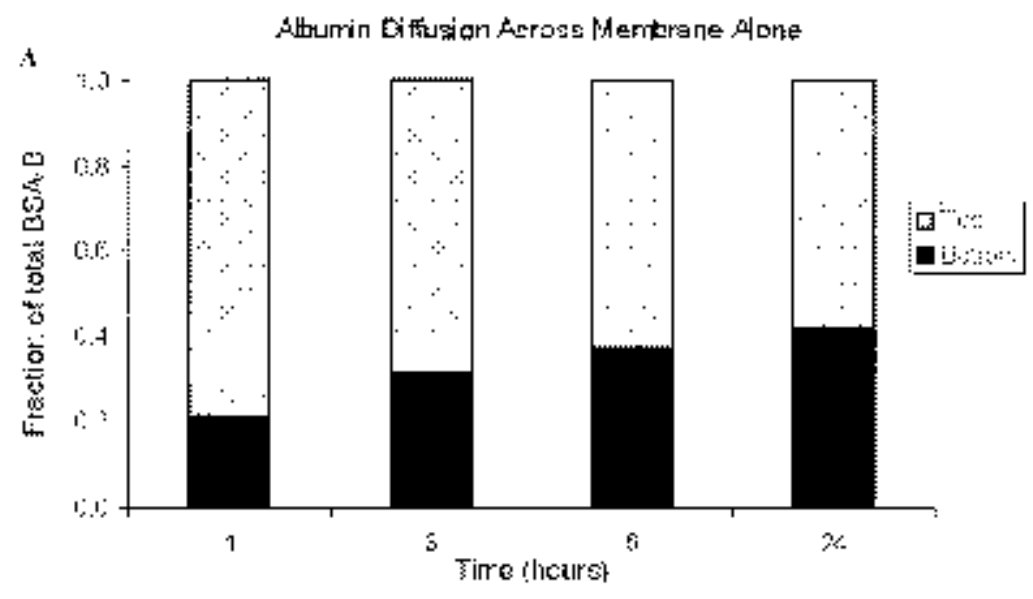

B. Asgumin Ciffusion Across Endathelial Cel: Monolayer

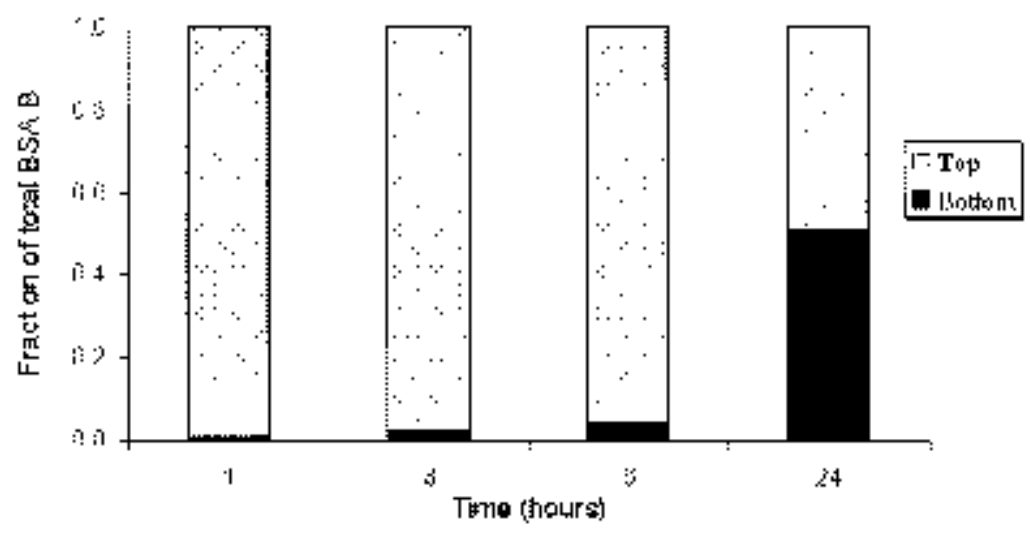

Figure 2. Albumin diffusion across membrane alone or membrane with confluent endothelial cell monolayers. BSA-biotin concentrations were measured in top and bottom compartments at $30 \mathrm{~min}, 2 \mathrm{~h}$, $4 \mathrm{~h}$, and $24 \mathrm{~h}$. Measurements from wells without cells (A) and with confluent HUVEC monolayers (B) were averaged, and values in each chamber were plotted as a percentage of the total BSA-biotin detected. 
(EBD)-protein conjugates are stable only for short periods, thereby necessitating repeated syntheses and lot-to-lot differences in labeling. This is to due to the tendency of Evans Blue-labeled proteins to form large polymeric com plexes at low temperature, thus making EBA an inferior marker for diffusion studies $(4,5)$. We have consistently observed prolonged stability of biotinBSA conjugate stored at $-80^{\circ} \mathrm{C}$ for up to nine months (unpublished data), allowing for delayed assessment of supernatant samples without degradation of marker integrity.

As with the other methods of determining monolayer leak, the greatest source of variability in this assay is in the integrity of the endothelial monolayer at the start of the experiment. This appears to be a problem unique to endothelial cell monolayers, as epithelial cells form tight barriers. The diameter of the semi-permeable membrane inserts used in this study was $12 \mathrm{~mm}$, and that of lower chambers was $22 \mathrm{~mm}$. In assays with new endothelial cell lines, we were able to estimate peak confluence solely by visual inspection on a phase contrast microscope and comparison of baseline biotin-BSA diffusion between cultures in unstimulated wells. Endothelial monolayer confluence was presumed to be adequate if values measured for these baseline wells were similar from culture to culture. Using these criteria for baseline monolayer integrity as a measure of confluence, inter-assay variability was minimized and was within the range of variability reported using fluorophore or radiolabeled reagents.

In summary, we present the use of biotinylated albumin followed by a rapid, plate-based detection with HRPstreptavidin as a sensitive, safe, reliable, and relatively easy means of assessing macromolecular diffusion across endothelial cell monolayers in vitro.

\section{REFERENCES}

\footnotetext{
1.Hofmann, K., S.W. Wood, C.C. Brinton, J.A. Montibeller, and F.M. Finn. 1980. Iminobiotin affinity columns and their application to retrieval of streptavidin. Proc. Natl. Acad. Sci. USA 77:4666-4668.

2.Horibe, Y., K. Hosoya, K.J. Kim, T. Ogiso, and V.H. Lee. 1997. Polar solute transport
}

across the pigmented rabbit conjunctiva: size dependence and the influence of 8-bromo cyclic adenosine monophosphate. Pharm. Res. 14:1246-1251.

3.Murphy, J.T., S. Duffy, G.F. Purdue, and J.L. Hunt. 1999. Thermal injury alters the endothelial vasoconstrictor and vasodilator response to endotoxin. J. Trauma 47:492-499.

4.Rawson, R.A. 1942. The binding of T-1824 and structurally related diazo dyes by the plasma proteins. Am. J. Physiol. 138:728-717.

5.Rogers, D.F., P. Boschetto, and P.J. Barnes. 1989. Plasma exudations correlation between Evan blue dye and radiolabeled albumin in guinea pig airways in vivo. J. Pharmacol. Methods 21:309-315.

6.Stanness, K.A., E. Guatteo, and D. Janigro. 1996. A dynamic model of the blood-brain barrier "in vitro". Neurotoxicology 17:481496.

7.Verin, A.D., F. Liu, N. Bogatcheva, T. Borbiev, M.B. Hershenson, P. Wang, and J.G. Garcia. 2000. Role of ras-dependent ERK activation in phorbol ester-induced endothelial cell barrier dysfunction. Am. J. Physiol. Lung Cell. Mol. Physiol. 279:L360-L370.

Address correspondence to Dr. Joseph T. Murphy, Department of Surgery, MC \#9158, 5323 H. Hines Blvd., University of Texas Southwestern Medical Center, Dallas, TX 75390-9158, USA. e-mail: joseph. Murphy@utsouthwestern.edu

Received 26 October 2000; accepted 28 March 2001.

Steve L. Duffy and Joseph T. Murphy

University of Texas

Southwestern Medical Center

Dallas, TX, USA

For reprints of this or any other article, contact Reprints@BioTechniques.com 